\title{
Triple negative breast cancer: A continuing challenge
}

Triple negative breast cancer (TNBC) is one of the few disease entities that is defined almost entirely by what it lacks, in this case, endocrine receptors and receptors for HER 2. This is also the reason why it has been so challenging to improve the outcomes in this subtype of breast cancer relative to others in which endocrine and targeted treatments have been successfully used. In contrast to Caucasian populations, the fraction of patients with TNBC is much higher in Asian populations, up to $25-30 \%$. Thus advances in the management of this disease are of particular interest to us.

A number of recent studies have shown that TNBC is not a homogeneous entity at the phenotypic and molecular levels. Clinically, this heterogeneity manifests itself in variable outcomes for patients treated with same or similar strategies. Although commonly considered to have poor prognosis, subgroups of TNBC patients, such as those who achieve pathological complete response after neoadjuvant chemotherapy, have excellent outcomes. Moreover, although they have a higher peak hazard of recurrence in the first few years after surgery as compared to patients with ER-positive tumors, women with TNBC who pass this landmark have a residual risk that is lower than the former. Thus, the view of TNBC as a homogeneous subtype with uniformly poor prognosis is an over-simplified one. There are other features that manifest this heterogeneity such as the association with young age, hereditary syndromes, propensity to visceral (including brain) metastases and poor post relapse survival in subgroups of TNBC patients. Pathologically, these tumors are diverse with a subgroup manifesting the typical features of high proliferation and grade, geographic necrosis, and lymphocytic infiltration.

The biggest advance in recent years has been the exquisite dissection of this heterogeneity at the molecular level. Several years ago, it was shown that breast cancers can be subdivided into so-called intrinsic subtypes using gene expression profiling patterns. The subgroup of "basal-like"

\begin{tabular}{|l|l|}
\hline \multicolumn{3}{|c|}{ Access this article online } \\
\hline Quick Response Code: & Website: \\
\hline & www.jmpo.org \\
\hline
\end{tabular}

cancers was shown to be about $70-80 \%$ concordant with the lack of expression of endocrine and HER2 receptors. Subsequently, it has been shown that a considerable fraction of these tumors express the basal cytokeratins (CK 5/6, CK 14) and epidermal growth factor receptor and have mutated p53. It has also became apparent that there is a complex relationship between breast tumors in patients carrying germline BRCA mutations, the majority of which have the TNBC and basal-like phenotypes and TNBC, only a minority of which carry germline mutation in these genes. More recently, it was shown that some TNBC, while not carrying the mutation, can nevertheless manifest "BRCAness" by other mechanisms such as epigenetic modulation of gene expression. Using next generation technologies that involve deep sequencing at genome, exome, and transcriptome levels, the extraordinary heterogeneity of TNBC has been dissected in a series of papers published in the last 2 years. Although it is beyond the scope of this piece to exemplify this in detail, it would be important to point out that the hallmark of typical basal-like, high-grade TNBC has been a very high level of genomic instability, a feature that they share and overlap with high-grade serous carcinoma of the ovary. Moreover, it is beginning to emerge that the larger picture is that of predominant loss of tumor suppression and scrambling of the genome, a difficult problem to surmount, rather than gain of tumor activation by oncogenes. It is hoped that a holistic synthesis of this new biological information will impact therapeutic strategies for TNBC in the near future.

At the clinical level, TNBC generally are chemo-sensitive tumors. It has been postulated that, because of their genomic instability, they may be particularly sensitive to DNA damaging agents such as alkylating agents and platinums. However, it has also become apparent that TNBC are probably sensitive to many different chemotherapy drugs, which may, at least partly, reflect higher proliferation in these tumors. Thus, the standard systemic treatment in the neo(adjuvant) setting remains anthracyclines and taxanes. There is some recent evidence from retrospective analysis of a Canadian adjuvant trial that $\mathrm{CMF}$ or a similar regimen may be particularly useful in TNBC, although this is by no means is definitively proven. Our Centre is currently engaged in a randomized phase 3 trial that is testing the value of adding carboplatin to a standard anthracycline-taxane regimen in the neoadjuvant setting in patients with TNBC. 
In the metastatic setting, a variety of agents including taxanes, platinums, gemcitabine, capecitabine, epothilones, vinca alkaloids, and others have been used singly or in combination. Unfortunately, the median survival is much lower in metastatic TNBC as compared to other subtypes. A number of new drugs have been evaluated in recent years, most conspicuously the PARP inhibitors that are based on the elegant concept of synthetic lethality. Although the definitive trial of one PARP inhibitor, iniparib, was negative in metastatic TNBC, PARP inhibition remains an active area of enquiry. Of note, tumors in patients with BRCA mutation have shown a much higher response to both PARP inhibition and platinums as compared to their non-mutated counterparts. These and other weaknesses of TNBC will hopefully be exploited in a rational manner to achieve better outcomes in the near future. Lastly, there is some evidence both from retrospective analyses and at least one prospective randomized trial that there is a lower threshold for benefit from adjuvant radiation in patients with TNBC, including after mastectomy.

Sudeep Gupta

Editor-in-Chief,

Professor of Medical Oncology, Breast Cancer Working Group, Tata Memorial Hospital, Mumbai, Maharashtra, India.

E-mail: editor@ijmpo.org

How to cite this article: Gupta S. Triple negative breast cancer: A continuing challenge. Indian J Med Paediatr Oncol 2013;34:1-2.

Announcement

\section{Android App}

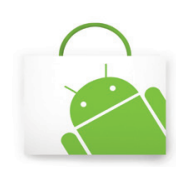

Download

Android application

A free application to browse and search the journal's content is now available for Android based mobiles and devices. The application provides "Table of Contents" of the latest issues, which are stored on the device for future offline browsing. Internet connection is required to access the back issues and search facility. The application is compatible with all the versions of Android. The application can be downloaded from https://market.android.com/details?id=comm.app.medknow. For suggestions and comments do write back to us. 